\title{
Auditorily elicited neural oscillations associated with motor execution, motor imagery and non-motor mental tasks
}

\author{
Horki $\mathrm{P}^{1}$, Klobassa DS ${ }^{1}$, Pokorny $\mathrm{C}^{1}$, and Müller-Putz $\mathrm{GR}^{1}$ \\ ${ }^{1}$ Institute for Knowledge Discovery, Graz University of Technology, Graz, Austria \\ petar.horki@tugraz.at
}

\begin{abstract}
In this work we investigated whether brisk feet dorsiflexion execution/imagery and a cognitive task related to working memory and perception of human voice elicited statistically significant event-related desynchronization/synchronization (ERDS) patterns in an auditory scanning paradigm. Following the auditory presentation of the target letter, seven out of ten participants displayed a central beta re-bound after brisk feet motor execution, whereas five out of ten participants also displayed similar, albeit weaker, ERDS patterns after imagination of the same movement. Six out of ten participants displayed frontal theta ERS in the non-motor cognitive task. Keywords: EEG, brain-computer interface (BCI), motor execution, motor imagery, working memory, auditory scanning.
\end{abstract}

\section{Introduction}

Auditory electroencephalogram (EEG)-based braincomputer interfaces (BCI) for spelling applications have mainly been pursued by means of event-related potentials $[1,2]$. However, spelling has also been successfully demonstrated by means of an event-related desynchronization/synchronization (ERDS) based BCI in the visual modality [3]. Furthermore, it was shown that ERDS can be used for communication both in visual [4] and binary choice auditory scanning mode [5]. Surprisingly, no attempts have been made towards ERDS based BCI spelling in auditory modality.

As a first step towards ERDS based BCI spelling in auditory modality, we investigated whether motor execution/imagery or a non-motor cognitive task could be detected in a multi-choice auditory scanning paradigm. To that end, we evaluated whether execution/imagery of brisk feet dorsiflexion $[6,7]$ and a cognitive task related to working memory $[8,9]$, and perception of human voice [10] elicited statistically significant ERDS patterns.

Our aim was to evaluate, if a multi-choice auditory scanning paradigm will lead to similar findings as previously described visually cued paradigms - i.e. if motor execution (ME) and motor imagery (MI) will lead to central ERS in the beta band $(15-40 \mathrm{~Hz})$, and that the cognitive task will lead to frontal ERS in the gamma band $(4-7 \mathrm{~Hz})$.

\section{Methods}

Ten healthy people ( 5 male, 5 female, college aged) participated in this experiment. Participants gave informed consent prior to the beginning of the experiments and received monetary compensation afterwards.
The EEG was recorded with 29 active electrodes overlying the frontal, central, and parietal scalp areas. The electrooculogram (EOG) was recorded with three active electrodes, and the electromyogram (EMG) with four electrodes from both legs. The EEG amplifiers were set up with a bandpass filter between 0.5 and $100 \mathrm{~Hz}$, and a notch filter at $50 \mathrm{~Hz}$. The EEG and EOG were sampled at $512 \mathrm{~Hz}$, the EMG at $2000 \mathrm{~Hz}$.

Spoken letters of the English alphabet, generated by a text-to-speech program, were presented sequentially (stimulus onset asynchrony $550 \mathrm{~ms}$ including $50 \mathrm{~ms}$ pause; $14.3 \mathrm{~s}$ for the whole alphabet) through a right headphone for one of several predefined words: "brain", "power", "husky" and "magic". Presenting acoustic cues through one ear only, keeps the other ear free for incoming communication from surroundings. For each target letter, the alphabet was presented two to four times.

The participants performed one of the following three tasks in the copy spelling mode: (1) brisk feet motor execution/imagery triggered by the target letter (ME/MI); (2) discrimination of the target voice's gender and comparison to the following repetition (i.e. whether the target voice's gender has changed or it remained the same, reporting through single/double button press) as a cognitive task (COG); and (3) mental repetition of the target letter as a control condition.

We balanced the order of motor and cognitive tasks, and voice of presentation. We randomized the order of words, and pseudorandomized the cognitive task and the control condition. Participants received no feedback.

Data analysis: We analyzed the central beta rebound in the ME/MI task and frontal theta band oscillations in the cognitive task.

To analyze the percentage of power decrease (ERD) or power increase (ERS) relative to a reference interval ( $0.5 \mathrm{~s}$ preceding the stimulus onset), a time-frequency map for frequency bands between 4 and $40 \mathrm{~Hz}$ (35 overlapping bands using a band width of $2 \mathrm{~Hz}$ ) was calculated. Logarithmic band power features, calculated by band-pass filtering, squaring and subsequently averaging over the trials, were used to assess changes in the frequency domain. To determine the statistical significance of the ERD/ERS values a t-percentile bootstrap algorithm with a significance level of $\alpha=0.01$ was applied. 
Table 1: Results of ERDS analysis for all participants. The ERDS analysis for ME and MI task was conducted on a single orthogonal Laplacian derivation centered at $\mathrm{Cz}$ electrode position, whereas analysis for COG task was conducted on a single bipolar derivation AFz-Fz. Shown here are only significant $(\mathrm{p}=0.01)$ results.

\begin{tabular}{|c|c|c|c|c|c|c|c|c|c|c|}
\hline Subj & S1 & $\mathrm{S} 2$ & S3 & $\mathrm{S} 4$ & S5 & S6 & S7 & S8 & S9 & $\mathrm{S} 10$ \\
\hline central $\beta$ ERS ME $_{\mathrm{ME}}$ & - & + & + & - & + & + & - & + & + & + \\
\hline central $\beta$-ERS MI $_{\mathrm{MI}}$ & - & + & + & - & + & + & - & - & - & + \\
\hline frontal $\vartheta$-ERS ${ }_{\mathrm{COG}}$ & + & - & + & - & + & + & + & - & - & + \\
\hline
\end{tabular}

\section{Results}

Seven out of ten participants displayed central beta rebound following brisk feet motor execution, whereas five out of ten participants also displayed similar, albeit weaker, ERDS patterns following imagination of the same movement (see Table 1). Six out of ten participants displayed frontal theta ERS in the cognitive task. Fig. 2 and Fig. 3 show examples of central beta ERS following brisk feet motor execution and of frontal theta ERS following the non-motor cognitive task, in one participant.

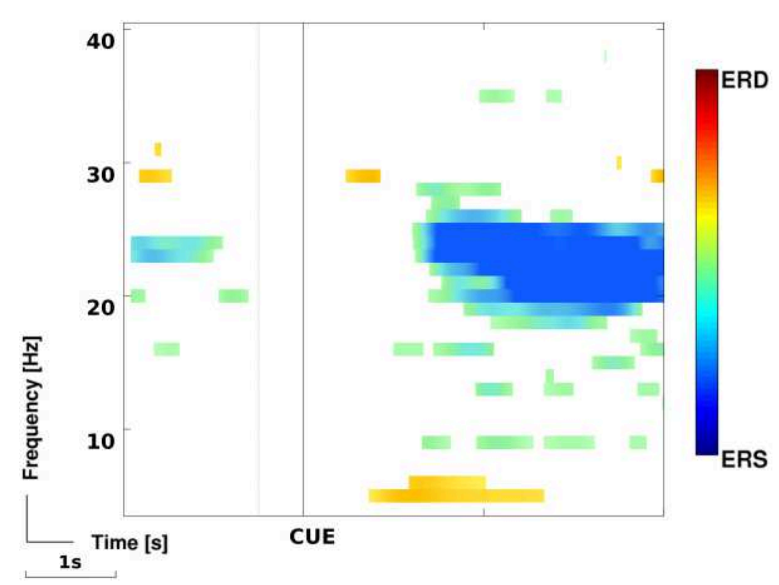

Figure 2: Central beta ERS following brisk feet motor execution in one participant.

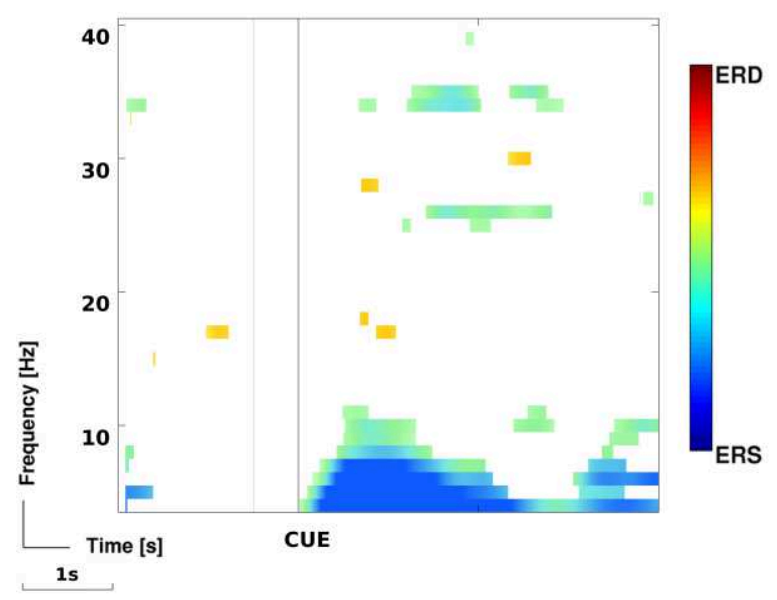

Figure 3: Frontal theta ERS following the COG task in one participant.

\section{Discussion}

The initial results indicate that task related ERDS changes in EEG could be exploited for auditory spelling. In future work we will investigate whether both ERDS and eventrelated potentials as combination can be used to determine which letter the participant was focusing on.

\section{Acknowledgement}

This research was supported by the European ICT Programme Project FP7-247919 (DECODER). This paper only reflects the authors' views and funding agencies are not liable. The authors would like to thank C. Breitwieser and M. Billinger for advice and assistance in the development of aspects of the BCI systems used in this work.

\section{Bibliography}

[1] Schreuder, M., Rost, T. et. al. Listen, You are Writing! Speeding up Online Spelling with a Dynamic Auditory BCI. Front. Neurosci., vol. 5, 2011

[2] Höhne, J., Schreuder, M. et. al. A novel 9-class auditory ERP paradigm driving a predictive text entry system. Front. Neurosci., vol. 5, 2011

[3] Scherer, R., Müller, G.R. et. al.: An asynchronously controlled EEG-based virtual keyboard: improvement of the spelling rate, IEEE Trans. Biomed. Eng., vol. 51, pp. 979-984, 2003

[4] Friedrich, E.V., McFarland, D.J. et. al. A scanning protocol for a sensorimotor rhythm-based brain-computer interface Biol. Psychol. vol.80, pp. 169-175, 2009.

[5] Müller-Putz, G.R., Pokorny, C. et. al. A single-switch BCI based on passive and imagined movements: towards restoring communication in minimally conscious patients Int. J. Neural Syst., vol. 23, 2013

[6] Pfurtscheller, G., Solis-Escalante, T.: Could the beta rebound in the EEG be suitable to realize a "brain switch"? Clin. Neurophysiol., vol. 120, pp. 24-29, 2009

[7] Müller-Putz, G.R., Kaiser, V. et. al.: Fast set-up asynchronous brain-switch based on detection of foot motor imagery in 1-channel EEG Med. Biol Eng. Comp., vol. 48, pp. 229-233, 2010

[8] Ruchkin, D.S., Johnson, R.et. al. Distinctions and similarities among working memory processes: an ERP study Cog. Brain Res., vol. 1, pp. 53-66, 1992.

[9] Klimesch, W., Doppelmayr, M. et. al. Theta synchronization during episodic retrieval: neural correlates of conscious awareness Cog. Brain Res., vol. 12, 2001

[10] Xu, H., Zhang, D. et al. Employing an active mental task to enhance the performance of auditory attentionbased brain-computer interfaces Clin. Neurophysiol., vol. 124, pp. 83-90, 2013 